\title{
Routine haemoglobin assay after uncomplicated caesarean sections
}

\author{
Ibrahim Abdelazim ${ }^{1,2}$, Mohamed Farghali ${ }^{1}$, Osama O. Amer ${ }^{3}$ \\ ${ }^{1}$ Department of Obstetrics, and Gynecology, Ain Shams University, Cairo, Egypt \\ ${ }^{2}$ Ahmadi Hospital, Kuwait \\ ${ }^{3}$ Ghamra Military Hospital, Cairo, Egypt
}

\begin{abstract}
Introduction: This study designed to detect whether the routine haemoglobin $(\mathrm{Hb})$ assay after uncomplicated caesarean section (CS) is necessary.

Material and methods: One hundred and twenty-two (122) women who delivered by uncomplicated elective CS were included in this observational study. Pre-operative investigations were performed according to the hospital protocol, including complete blood count, haemoglobin, prothrombin time, activated partial thromboplastin time, and liver and kidney function tests. After the uncomplicated elective CS, blood samples taken from participants immediately, 12, 24, 48 hours, and 1-week post-operative (PO) for haemoglobin assay. Student's $t$-test was used to compare the pre-operative, and PO haemoglobins to detect whether or not the Hb assay after uncomplicated CSs is necessary.

Results: There was no significant difference between the pre-operative haemoglobin (11.6 $\pm 6.4 \mathrm{gms} \%)$, and the immediate PO haemoglobin (11.1 $\pm 5.9 ; p=0.1$ [95\% Cl: $-1.05,0.5,2.05])$ or 12-hour PO haemoglobin (10.9 $\pm 7.3 ; p=0.9[95 \% \mathrm{Cl}:-1.03,0.7,2.43])$ or 24 -hour PO haemoglobin $(10.7 \pm 8.2 ; p=0.9[95 \% \mathrm{Cl}:-0.95,0.9,2.75])$. In addition, there was no significant difference between the pre-operative haemoglobin (11.6 \pm 6.4 gms\%), and 48-hour PO haemoglobin (11.2 $\pm 6.9 ; p=0.7$ [95\% Cl: $-1.28,0.4,2.08])$, or 1-week PO haemoglobin (11.4 \pm 7.5 ; $p=0.9[95 \% \mathrm{Cl}:-1.55,0.2,1.95])$.

Conclusions: Routine PO haemoglobin assay after uncomplicated elective CSs is not necessary, especially when the pre-operative haemoglobin before the ECS $\geq 11 \mathrm{gms} \%$, CS duration < $45 \mathrm{~min}$, and estimated intraoperative blood loss $\leq 500 \mathrm{~mL}$.
\end{abstract}

Key words: postpartum, haemoglobin, uncomplicated, elective, caesarean section.

\section{Introduction}

Caesarean section (CS) is the commonest surgical procedure in obstetrics. Blood loss and blood transfusion are major complications of CS $[1,2]$.

The massive uterine hyper-perfusion $(750 \mathrm{~mL} / \mathrm{min})$ in late pregnancy (10-12\% of cardiac output) [3] explains the high blood loss during CS (500-750 mL) [4].

Women delivering by CS are at higher risk of postpartum haemorrhage than those delivering vaginally $[5,6]$.

The blood loss during CS is usually underestimated by the surgeon [7]. Underestimated blood loss during CS exposes women to the risk of uncorrected blood loss and uncorrected anaemia, particularly poor wound healing, surgical site infection (SSI), fatigue, and physical disability $[8,9]$.

Routine haemoglobin $(\mathrm{Hb})$ assay is one of the common post-operative (PO) practices after CS [10], which exposes women to unnecessary blood components transfusion and transfusion risks due to misdiagnosed anaemia [11]. Therefore, this study aimed to detect whether or not $\mathrm{Hb}$ assay after uncomplicated CSs is necessary.

\section{Material and methods}

This observational study was conducted over 1 year, from December 2018 to December 2019, after approval of the Obstetrics and Gynaecology Department Ethical Committee, and obtaining informed consent in accordance with the Declaration of Helsinki.

One hundred and twenty-two (122) women delivering by uncomplicated elective CS (ECS) were included in this study to detect whether or not the $\mathrm{Hb}$ assay after CS is necessary.

Inclusion criteria included women between 20 and 40 years old, 1) admitted for ECS for the first delivery due to maternal request [12] or abnormal presenting foetal part (breech presentation), or 2) admitted for elective repeat CS (ERCS) following refusal of trial of la- 
bour after 1 previous CS $[13,14]$, or 3 ) admitted for ERCS following a previous 2 or 3 CSs.

Women with anaemia (haemoglobin < $10 \mathrm{gms} \%$ ), medical disorders with pregnancy (hypertension, preeclampsia, and/or diabetes), uterine overdistension (foetal macrosomia, twin pregnancy, polyhydramnios), ante-partum haemorrhage, placenta previa, bleeding tendency, coagulation disorders, anticoagulant therapy, risks of thrombo-embolism, and/or post-partum haemorrhage were excluded from this study.

Uncomplicated CS was defined when the duration of the CS was $<45 \mathrm{~min}$ with $<750 \mathrm{~mL}$ intra-operative blood loss, no bladder, ureteral, intestinal, or uterine artery injuries, and no uterine atonia [10].

Participants were subjected to thorough history, abdominal examination, and abdominal ultrasound for assessment of the foetal position, amniotic fluid, and placental site, followed by pre-operative investigations according to the hospital protocol (complete blood count, haemoglobin, prothrombin time, activated partial thromboplastin time, liver, and kidney function tests).

All CSs were done under spinal anaesthesia, by the obstetrics and a gynaecology consultant ( $\geq 15$ years' experience after MD degree), assisted by the registrar ( $\geq 8$ years' experience after a master's degree).

After a Joel-Cohen incision, which has better PO outcome than Pfannenstiel incision [15], the anterior abdominal wall was opened in layers, followed by opening of the visceral peritoneum above the lower uterine segment (LUS) to create the bladder flap and to dissect the urinary bladder away from the LUS [16].

A transverse uterine incision was done in LUS, then the amniotic fluid (AF) was drained by suction to minimize soaking of the towels and gauzes by the AF as possible. After draining of the AF and delivery of the foetus, the umbilical cord was clamped and $20 \mathrm{IU}$ of oxytocin (Syntocinon, Novartis Pharmaceutical, Egypt) intravenous over $500 \mathrm{~mL}$ of normal saline at an infusion rate of $125 \mathrm{~mL} / \mathrm{h}$ was given as an active management of the third stage of labour, and to avoid uterine atony [17].

After spontaneous delivery of the placenta, the uterine incision was repaired in 2 layers using no. 1 absorbable polyglycolic sutures (Vicryl-Ethicon, NJ, USA) [10].

The blood loss during CS was estimated according to the amount of blood in the suction bottle and the number of soaked towels (soaked towel $=150 \mathrm{~mL}$, while semi-soaked towel $=75 \mathrm{~mL}$ ) by the anaesthetist who attended the CS (to avoid the surgeon`s bias) [18]. In addition, the blood loss during CS was confirmed by the weight difference of the used towels during CS (PO weight of the used towels during CS - preoperative weight). An accurate digital weighing scale was used, and each $1 \mathrm{~g}$ difference in weight corresponds to $1 \mathrm{~mL}$ of blood loss [19]. Blood samples were taken from participants immediately, 12, 24, and 48 hours, and 1 week PO for haemoglobin assay. Student's t-test was used to compare the pre-operative and post-operative haemoglobin levels to detect whether or not the $\mathrm{Hb}$ assay after uncomplicated CS is necessary (primary outcome). Secondary outcome measures were the relationship between haemoglobin drop after CS, and intraoperative blood loss, or CS duration.

\section{Sample size justification}

The required sample size was calculated using G Power software version 3.17 for sample size calculation, setting $\alpha$ error probability at 0.05 , power (1- $\beta$ error probability) at $0.95 \%$, and effective sample size (w) at 0.5 . An effective sample size $\geq 110$ women was needed to produce a statistically acceptable figure.

\section{Statistical analysis}

Collected data were statistically analysed using Statistical Package for Social Sciences: computer software version 20 (Chicago, IL, USA). Numerical variables were presented as mean and standard deviation ( \pm SD), while categorical variables were presented as number $(n)$ and percentage (\%). Student's $t$-test was used to compare the pre-operative haemoglobin with post-operative haemoglobins, to detect whether or not the $\mathrm{Hb}$ assay after uncomplicated CS is necessary. Pearson's correlation coefficient was used to evaluate the relationship between the haemoglobin drop after the CS and intraoperative blood loss or CS duration. $p<0.05$ was considered significant.

\section{Results}

One hundred and twenty-two (122) women who delivered by uncomplicated ECS were included in this observational study. Pre-operative haemoglobin was compared with haemoglobin collected the immediately, 12,24 , and 48 hours, and 1 week PO to detect whether or not the $\mathrm{Hb}$ assay after uncomplicated CS is necessary.

The mean age of the studied women was 24.1 \pm 5.6 years, mean gestational age at ECS was 39.2 \pm 1.2 weeks, and mean body mass index (BMI) was 26.6 $\pm 3.4 \mathrm{~kg} / \mathrm{m}^{2}$. The ECS was primi-CS in $22.1 \%$ (27/122), and it was ERCS in $77.9 \%$ (95/122) of the studied women. The mean pre-operative haemoglobin of the studied women was $11.6 \pm 6.4 \mathrm{~g} / \mathrm{dL}$, mean operative time was $43.1 \pm 7.2 \mathrm{~min}$, and mean estimated intra-operative blood loss was $485.9 \pm 136.7 \mathrm{~mL}$ (Table 1 ).

There was no significant difference between the pre-operative haemoglobin (11.6 $\pm 6.4 \mathrm{gms} \%)$, and the immediate PO haemoglobin (11.1 $\pm 5.9 ; p=0.1$ [95\% Cl: $-1.05,0.5,2.05])$ or 12 -hour PO haemoglobin (10.9 \pm 7.3 ; $p=0.9$ [95\% Cl: $-1.03,0.7,2.43])$ or 24 -hour PO haemoglobin $(10.7 \pm 8.2 ; p=0.9$ [95\% Cl: $-0.95,0.9,2.75])$. In 
addition, there was no significant difference between the pre-operative haemoglobin (11.6 $\pm 6.4 \mathrm{gms} \%$ ), and 48-hour PO haemoglobin (11.2 $\pm 6.9 ; p=0.7$ [95\% Cl: $-1.28,0.4,2.08])$, or 1 -week PO haemoglobin (11.4 \pm 7.5 ; $p=0.9$ [95\% Cl: $-1.55,0.2,1.95]$ ) (Table 2).

The mean haemoglobin drop after CS was $0.54 \pm 7.1$ gms\%, mean intra-operative blood loss during CSs was $485.9 \pm 136.7 \mathrm{~mL}$, and mean CS operative time was 43.1 $\pm 7.2 \mathrm{~min}$. No strong positive correlation found between the haemoglobin drop after the CS and intra-operative blood loss ( $r=0.22$ [weak positive correlation]) (Fig. 1) or CSs duration ( $r=0.69$ [moderate positive correlation]) (Fig. 2) using Pearson's correlation coefficient.

\section{Discussion}

Routine haemoglobin assay is one of the common PO practices after CS [10], which exposes women to unnecessary blood component transfusion for misdiagnosed anaemia [11]. Therefore, this study was designed to detect whether or not the $\mathrm{Hb}$ assay after uncomplicated CSs is necessary.

Therefore, 122 women who delivered by uncomplicated ECSs were included in this study, and the pre-operative haemoglobin of the studied women was compared with haemoglobin collected immediately, 12, 24, and 48 hours, and 1 week PO, to detect whether or not the $\mathrm{Hb}$ assay after uncomplicated CSs is necessary.

The mean age of the studied women was 24.1 \pm 5.6 years, mean gestational age at ECS was 39.2 \pm 1.2 weeks, and mean BMI was $26.6 \pm 3.4 \mathrm{~kg} / \mathrm{m}^{2}$. The ECS was primi-CS in $22.1 \%$ (27/122), and it was ERCS in $77.9 \%(95 / 122)$ of the studied women. The mean pre-operative haemoglobin of the studied women was $11.6 \pm 6.4 \mathrm{~g} / \mathrm{dL}$, mean operative time was $43.1 \pm 7.2 \mathrm{~min}$, and mean estimated intra-operative blood loss was $485.9 \pm 136.7 \mathrm{~mL}$.

Bodur et al. [10] and other obstetricians prefer to do the ECS 38 weeks after the dexamethasone course
Table 1. Demographic data, type of elective caesarean sections, pre-operative haemoglobin, operative time, and intraoperative blood loss of the studied women

\begin{tabular}{lc}
\hline Variables & $\begin{array}{c}\text { Studied women } \\
(n=122)\end{array}$ \\
\hline Maternal age (years) & $24.1 \pm 5.6$ \\
\hline Gestational age at ECS (weeks) & $39.2 \pm 1.2$ \\
\hline BMI (kg/m²) & $26.6 \pm 3.4$ \\
\hline Type of the ECS & $27 / 122(22.1 \%)$ \\
\hline Primi-ECS & $95 / 122(77.9 \%)$ \\
\hline ERCS & $11.6 \pm 6.4$ \\
\hline Pre-operative haemoglobin $(\mathrm{gm} / \mathrm{dL})$ & $43.1 \pm 7.2$ \\
\hline Operative time $(\mathrm{min})$ & $485.9 \pm 136.7$ \\
\hline Estimated intra-operative blood loss $(\mathrm{mL})$ & \\
\hline
\end{tabular}

$\mathrm{BMI}$ - body mass index, ECS - elective caesarean section, ERCS - elective repeat caesarean section

Data presented as number and percentage (\%), and mean \pm standard deviation (SD).

for induction of foetal lung maturity [20]. In this study, the mean gestational age at ECS was $39.2 \pm 1.2$ weeks, which can be explained by the ACOG guidelines [21].

ACOG recommends delaying ECS at spontaneous labour pains to allow oxytocin release, and its beneficial effect on epithelial sodium channels (ENaC), which prepares the lung for gas exchange, and reduces the neonatal respiratory morbidity [21]. In addition, Abdelazim et al. found significant reduction in neonatal respiratory morbidity when the ECS is performed at $\geq 39$ weeks of gestation [21].

The incidence of primi-CS in this study was $22.1 \%$ (27/122), which can be explained by increased incidence of primi-CS all over the world, due to maternal preferences, improved safety of anaesthesia and blood products, and extensive foetal monitoring [22].

The World Health Organization recommends that the CS rate should not exceed $10-15 \%$ of all deliveries in any country [23]. The CS rate in most Middle East countries is $<15 \%$, and the highest CS rates are reported in

Table 2. Pre-operative haemoglobin compared to the immediate, 12-, 24-, 48-hour, and 1-week post-operative haemoglobin levels

\begin{tabular}{lcc}
\hline Variables & Studied women $(n=122)$ & $p$-value (95\% Cl) \\
\hline Pre-operative haemoglobin (gms\%) & $11.6 \pm 6.4$ & $11.1 \pm 5.9$ \\
Immediate PO haemoglobin (gms\%) & $11.6 \pm 6.4$ & $0.1(-1.05,0.5,2.05)$ \\
\hline Pre-operative haemoglobin (gms\%) & $10.9 \pm 7.3$ & $0.9(-1.03,0.7,2.43)$ \\
12-hrs PO haemoglobin (gms\%) & $11.6 \pm 6.4$ & $0.9(-0.95,0.9,2.75)$ \\
\hline Pre-operative haemoglobin (gms\%) & $10.7 \pm 8.2$ & $0.7(-1.28,0.4,2.08)$ \\
24-hrs PO haemoglobin (gms\%) & $11.6 \pm 6.4$ \\
\hline Pre-operative haemoglobin (gms\%) & $11.2 \pm 6.9$ & \\
48-hrs PO haemoglobin (gms\%) & $11.6 \pm 6.4$ & $0.9(-1.55,0.2,1.95)$ \\
\hline Pre-operative haemoglobin (gms\%) & $11.4 \pm 7.5$ & \\
1-week PO haemoglobin (gms\%) & & \\
\hline
\end{tabular}

$\mathrm{Cl}$ - confidence interval, hrs - hours, $\mathrm{PO}$ - post-operative

Data presented as mean \pm standard deviation.

Student $t$-test used for statistical analysis. 


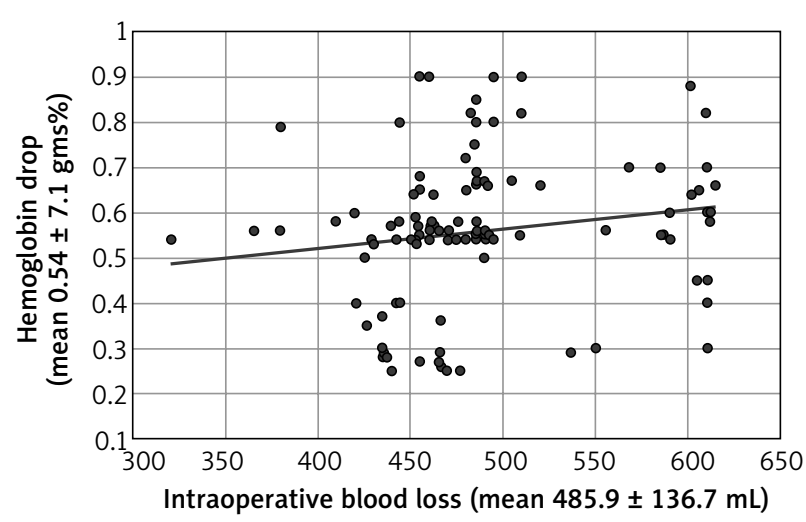

Fig. 1. Correlation between haemoglobin drop after caesarean section and intraoperative blood loss during caesarean section

Egypt (26\%) and Sudan (20\%) [22]. Tapia et al. reported that the CS rate was $27 \%$ in 3 Peruvian regions between 2000 to 2010 , with significant yearly increase in the CS rate from $23.5 \%$ to $30 \%$ [24].

In the United States, the CS rate reached 30\% in 2006. In Europe, the CS rate varies from $52.2 \%$ in Cyprus to $14.8 \%$ in Iceland, and $24.6 \%$ in England. In addition, an increase in the CS rate was observed in China, India, Nepal, and Bangladesh [23].

The mean operative time of the uncomplicated CSs in this study was $43.1 \pm 7.2 \mathrm{~min}$. Also, Bodur et al. defined the CS as uncomplicated when the duration was no longer than $45 \mathrm{~min}$ and there were no bladder, ureteral, intestinal, uterine artery injuries, or uterine atonia [10].

In addition, Tuuli et al. studied 258 women undergoing primi-ECS and ERCS $\geq 32$ weeks to compare the operative time during creation $(n=131)$ or omission ( $n=127$ ) of the bladder flap. They found that the median operative time was similar, with no significant difference in the 2 studied groups (51 $\mathrm{min}$ ) [17].

The mean estimated blood loss in this study for uncomplicated ECS, whether primi-CS or ERCS, was 485.9 $\pm 136.7 \mathrm{~mL}$. Similarly, Bodur et al. found that the average estimated intra-operative blood loss during uncomplicated CS was $517.06 \pm 417.55 \mathrm{~mL}$ for all CSs $(535.60$ $\pm 475.23 \mathrm{~mL}$ for previous CS vs. $501.24 \pm 365.20 \mathrm{~mL}$ for primi-CS) [10].

An average blood loss of $\leq 750 \mathrm{~mL}$ during surgery is considered acceptable by the American College of Surgeons, while $>750 \mathrm{~mL}$ average blood loss is considered as Class-I haemorrhage in which minimal physiological changes occur [10, 25].

Qatawneh et al. reviewed cases managed by emergency peripartum hysterectomy (EPH) and found that the estimated blood loss during EPH was $2210 \mathrm{~mL}, 17.6 \%$ of their studied women bled $>3000 \mathrm{cc}$, and the average number of blood units transfused was $3.93 \pm 3.43$ [26].

In this study, there was no significant difference between the pre-operative haemoglobin, and the im-

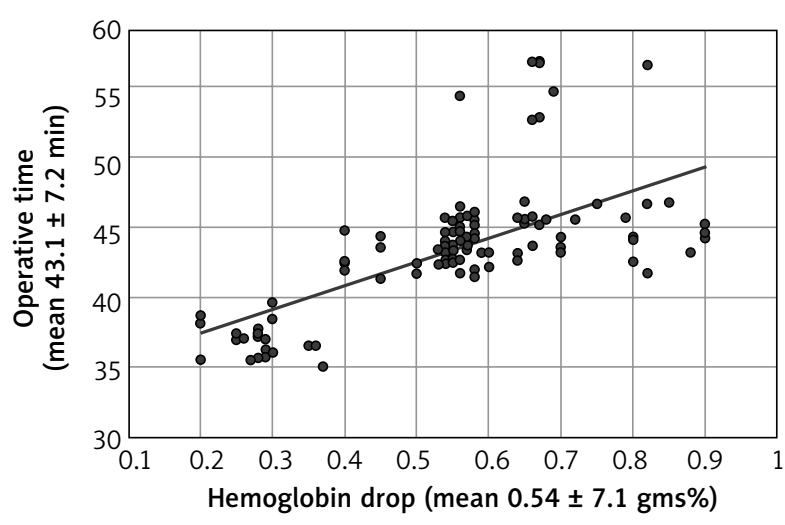

Fig. 2. Correlation between haemoglobin drop after caesarean section and caesarean section duration

mediate or 12-hour or 24-hour PO haemoglobin ( $p=0.1$, 0.9 , and 0.9 , respectively). In addition, there was no significant difference between the pre-operative haemoglobin, and 48-hour or 1-week PO haemoglobin ( $p=0.7$ and 0.9 , respectively) after uncomplicated ECS.

Carson et al. used the 1-week PO haemoglobin to evaluate the blood transfusion strategies in high-risk patients after hip surgery [27], and Singh et al. used the 2-day postpartum haemoglobin assay to evaluate the post-CS haemoglobin change and/or drop [28].

Anzaku et al. found that routine post-CS haematocrit testing is unnecessary following uncomplicated CS, but it may be indicated in women with preoperative haematocrit $\leq 35 \%$, PO fatigue, and conjunctival pallor [29].

Horowitz et al. concluded that routine PO haemoglobin assay after an uncomplicated elective CS in asymptomatic low-risk women is not necessary [30]. In addition, Api et al. concluded that $\mathrm{Hb}$ testing following uneventful CS neither changed the PO management nor determined women requiring blood transfusion [31].

No strong positive correlation was found between the haemoglobin drop after the CS and intra-operative blood loss during CS ( $r=0.22$ [weak positive correlation]), or CS in this study. Also, Singh et al. found weak positive correlation between the haemoglobin drop after CS and operative duration from uterine incision till repair [28].

This study concluded that the routine PO haemoglobin assay after uncomplicated elective CS is not necessary, especially when the pre-operative haemoglobin before the ECS $\geq 11$ gms\%, CS duration < $45 \mathrm{~min}$, and the estimated intra-operative blood loss $\leq 500 \mathrm{~mL}$.

The current study was the first observational study conducted in Kuwait, and it concluded that the $\mathrm{Hb}$ assay after uncomplicated elective CSs is not necessary. Routine practice of PO haemoglobin assay after uncomplicated CS should be stopped.

Women who refused to give consent and/or participate, and the absence of a control group were the limitations faced during this study. 


\section{Conclusions}

Routine PO haemoglobin assay after uncomplicated elective CS is not necessary, especially when the pre-operative haemoglobin before the ECS $\geq 11$ gms $\%$, CS duration $<45 \mathrm{~min}$, and the estimated intra-operative blood loss $\leq 500 \mathrm{~mL}$.

\section{Acknowledgements}

The authors are grateful to the women who agreed and gave consent to participate in this study.

The study was approved by the Obstetrics and Gynaecology Department Ethical Committee. Informed consent was obtained from the studied women in accordance with the Declaration of Helsinki.

\section{Disclosure}

The authors report no conflict of interest.

\section{References}

1. Ramadani H. Cesarean section intraoperative blood loss and mode of placental separation. Int J Gynaecol Obstet 2004; 87: 114-8.

2. Murphy DJ, MacGregor H, Munishankar B, et al. A randomised controlled trial of oxytocin $5 I U$ and placebo infusion versus oxytocin $5 I \mathrm{U}$ and $30 \mathrm{IU}$ infusion for the control of blood loss at elective caesarean section pilot study. ISRCTN 40302163. Eur J Obstet Gynecol Reprod Biol 2009; 142: 30-33.

3. Flo K, Wilsgaard T, Vårtun A, et al. A longitudinal study of the relationship between maternal cardiac output measured by impedance cardiography and uterine artery blood flow in the second half of pregnancy. BJOG 2010; 117: 837-844.

4. Stafford I, Dildy GA, Clark SL, et al. Visually estimated and calculated blood loss in vaginal and cesarean delivery. Am J Obstet Gynecol 2008; 199: 519.e1-7.

5. Al-Zirqi I, Vangen S, Forsen L, et al. Prevalence and risk factors of severe obstetric haemorrhage. BJOG 2008; 115: 1265-1272.

6. Bateman BT, Berman MF, Riley LE, et al. The epidemiology of postpartum hemorrhage in a large, nationwide sample of deliveries. Anesth Analg 2010; 110: 1368-1373.

7. Schorn MN. Measurement of blood loss: review of the literature. J Midwifery Womens Health 2010; 55: 20-27.

8. Milman N. Postpartum anemia II: prevention and treatment. Ann Hematol 2012; 91: 143-154.

9. Lagoo J, Wilkinson J, Thacker J, et al. Impact of anemia on surgical outcomes: innovative interventions in resource-poor settings. World J Surg 2012; 36: 2080-2089.

10. Bodur S, Gun I, Ozdamar O, et al. Safety of uneventful cesarean section in terms of hemorrhage. Int J Clin Exp Med 2015; 8: 21653-2168.

11. Shander A, Goodnough LT. Why an alternative to blood transfusion? Crit Care Clin 2009; 25: 261-277.

12. Olieman RM, Siemonsma F, Bartens MA, et al. The effect of an elective cesarean section on maternal request on peripartum anxiety and depression in women with childbirth fear: a systematic review. BMC Pregnancy Childbirth 2017; 17: 195.

13. Abdelazim IA, Elbiaa AA, Al-Kadi M, et al. Maternal and obstetrical factors associated with successful trial of vaginal birth after caesarean section. J Turk Ger Gynecol Assoc 2014; 15: 245-249.

14. Sakiyeva KZ, Abdelazim IA, Farghali M, et al. Outcome of the vaginal birth after cesarean section during the second birth order in West Kazakhstan. J Family Med Prim Care 2018; 7: 1542-1547.

15. Mathai M, Hofmeyr GJ, Mathai NE. Abdominal surgical incisions for caesarean section. Cochrane Database Syst Rev 2013; (5): CD004453.
16. Walsh CA. Evidence-based cesarean technique. Curr Opin Obstet Gynecol 2010; 22: 110-115.

17. Tuuli MG, Odibo AO, Fogertey P, et al. Utility of the bladder flap at cesarean delivery: a randomized controlled trial. Obstet Gynecol 2012; 119: 815-821.

18. Yehia AH, Koleib MH, Abdelazim IA, et al. Tranexamic acid reduces blood loss during and after cesarean section: a double blinded, randomized, controlled trial. Asian Pac J Reprod 2014; 3: 53-56.

19. Kathariya $\mathrm{R}$, Devanoorkar A, Jain $\mathrm{H}$. Intra-operative hemorrhage: a review of literature. J Med Diagn Meth 2013; 2: 6.

20. Abdelazim IA, Alanwar A, Svetlana S, et al. Complications associated with higher order compared to lower order cesarean sections. J Matern Fetal Neonatal Med 2020; 33: 23952402.

21. Abdelazim I, Farghali MMM, Elbiaa AAM, et al. Impact of antenatal oxytocin infusion on neonatal respiratory morbidity associated with elective cesarean section. Arch Med Sci 2017; 13: 629-634.

22. Abdelazim IA, Alanwar A, Svetlana S, et al. Complications associated with higher order compared to lower order cesarean sections. J Matern Fetal Neonatal Med 2020; 33: 23952402.

23. Bhatia M, Banerjee K, Dixit P, et al. Assessment of variation in cesarean delivery rates between public and private health facilities in india from 2005 to 2016. JAMA Netw Open 2020; 3: e2015022.

24. Tapia V, Betran AP, Gonzales GF. Caesarean section in Peru: analysis of trends using the Robson Classification System. Plos ONE 2016; 11: e0148138.

25. Kortbeek JB, Al Turki SA, Ali J, et al. Advanced trauma life support, 8th edition, the evidence for change. J Trauma 2008; 64: 1638-1650.

26. Qatawneh A, Fram K, Thikerallah F, et al. Emergency peripartum hysterectomy at Jordan University hospital - a teaching hospital experience. Menopause Rev 2020; 19: 66-71.

27. Carson JL, Terrin ML, Noveck H, et al. Liberal or restrictive transfusion in high-risk patients after hip surgery. N Engl J Med 2011; 365: 2453-2462.

28. Singh B, Adhikari N, Ghimire S, et al. Post-operative drop in hemoglobin and need of blood transfusion in cesarean section at Dhulikhel Hospital, Kathmandu University Hospital. Kathmandu Univ Med J (KUMJ) 2013; 11: 144-146.

29. Anzaku AS, Edem BE, Ngwan SD, et al. Do patients require routine hematocrit testing following uncomplicated cesarean delivery? Afr J Med Health Sci 2015; 14: 24-28.

30. Horowitz E, Yogev Y, Ben-Haroush A, et al. Routine hemoglobin testing following an elective cesarean section: is it necessary? J Matern Fetal Neonatal Med 2003; 14: 223-225.

31. Api O, Unal O, Api M, et al. Do asymptomatic patients require routine hemoglobin testing following uneventful, unplanned cesarean sections? Arch Gynecol Obstet 2010; 281: 195-199. 